\title{
So klappt die Kommunikation
}

\begin{abstract}
Die Mitteilung einer schwerwiegenden Diagnose wie Parkinson belastet Arzt wie Patient. Das Bedürfnis der Patienten nach Informationen ist oft groß, die Kommunikation gelingt aber nicht immer verständlich und situationsgerecht. Ein Kommunikationsworkshop in Hamburg zeigte Fallstricke und Lösungsmöglichkeiten auf.

„Am Anfang wird die Basis gelegt für die gegenseitigen Erwartungen von Arzt und Patient", betonte Diplompsychologe Dr. Andreas Loh, Universitätsklinikum Freiburg. Das stresst Ärzte oft ebenso wie Patienten. Einerseits sind die Diagnose und Informationen zur Erkrankung zu übermitteln, andererseits ist es wichtig, den Emotionen und Bedürfnissen des $\mathrm{Pa}$ tienten Raum zu geben und ein Vertrauensverhältnis zu schaffen. Dabei helfen Kommunikationsmethoden wie das verständnisvolle Zuhören, das Paraphrasieren und das Verbalisieren emotionaler Erlebnisinhalte.
\end{abstract}

\section{Zeit nehmen}

Das alles braucht Zeit, doch die macht sich auf Dauer bezahlt. Professor Wolfgang Jost von der Deutschen Klinik für Diagnostik in Wiesbaden erklärt beispielsweise seinen Patienten auch Differenzialdiagnosen. „Wenn ich das nicht tue, lesen die Patienten es im Internet“, sagt er. Er erläutert Patienten, warum bestimmte Diagnoseverfahren je nach Situation keinen Sinn machen, zum Beispiel ein DAT-Scan bei eindeutigen klinischen Befunden, und warum Nahrungsergänzungsmittel meist wenig Sinn machen.

Die neuen DGN-Leitlinien haben die Anforderung an die Aufklärung zudem erweitert - auf das Risiko von Impulskontrollstörungen bei Therapie mit Dopaminagonisten sollte - auch aus haftungsrechtlichen Gründen - explizit hingewiesen werden. Neu ist zudem die Empfehlung eines Therapiebeginns direkt nach Diagnosestellung. In Studien trat bei Frühtherapie die Progression in ein Hoehn \& Yahr-Stadium IV und V später ein, die Lebensqualität blieb länger erhalten. Im Rahmen der medikamentösen Therapie haben retardierte Dopaminagonisten, wie zum Beispiel retardiertes Pramipexol $\left(\right.$ Sifrol $\left.^{\oplus}\right)$, Vorteile, so Jost: eine bessere Compliance bei einmal täglicher Gabe, gleichmäßigere Wirkspiegel, weniger Nebenwirkungen und einen besseren Schlaf. Schon in der Frühphase muss die Therapie aber multimodal erfolgen, das heißt Beratung, medikamentöse und nicht-medikamentöse Maßnahmen müssen aufeinander abgestimmt werden und auch nicht-motorische Störungen sind zu berücksichtigen, forderte Jost.

Friederike Klein, freie Medizinjournalistin

Kommunikationsworkshop für Neurologen "Patientenzentriertes Parkinsonmanagement Gesprächsführung und erfolgreicher Therapiestart", 85. Kongress der Deutschen Gesellschaft für Neurologie, Hamburg, 27.9.2012 Veranstalter: Boehringer Ingelheim

\section{Zulassung neuer oraler Basistherapie wahrscheinlich}

Zwei Phase-III-Studien mit BG-12 (Dimethylfumarat) zur Therapie der schubförmigen Multiplen Sklerose (MS) haben eine gute Wirksamkeit hinsichtlich der Reduktion der Schubrate, der Behinderungsprogression und der Krankheitsparameter in der Bildgebung gezeigt. Eine gepoolte Analyse dieser Daten wurde jetzt auf dem ECTRIMS vorgestellt.

Diese Analyse war im Vorhinein festgelegt und spezifiziert worden, betonte Professor Ralf Gold, Direktor der neurologischen Klinik am St. Josef-Hospital der Ruhr-Universität Bochum. Das war möglich, weil beide Studien ein identisches Design hatten. DEFINE (Determination of the Efficacy and Safety of Oral Fumarate) [Gold R et al. N Engl J Med. 2012; 367: 1098-107] and CONFIRM (Comparator and an Oral Fumarate in RRMS) [Fox RJ et al. N Engl J Med. 2012; 367: 1087 -97] waren randomisierte Doppelblindstudien zur Untersuchung von
Wirksamkeit und Sicherheit von Dimethylfumarat (240 mg), entweder zweimal oder dreimal täglich eingenommen, im Vergleich zu Placebo. CONFIRM beinhaltete außerdem als Referenz einen Arm, in dem Patienten eine Therapie mit Glatirameracetat (20 mg s.c. täglich) erhielten. Die gepoolten Daten von insgesamt 2.301 Patienten sollen die Sicherheit bezüglich der Wirksamkeit und unerwünschten Wirkungen von Dimethylfumarat weiter verbessern.

Im Vergleich zu Placebo reduzierte die Therapie mit Dimethylfumarat danach die Schubrate um etwa die Hälfte gegenüber Placebo (49\%, p < 0,0001). Das Schubrisiko sank damit im Vergleich zu Placebo um $43 \%$ ( $<<0,0001)$. Das Risiko, eine anhaltende Behinderung zu entwickeln, war in der Verumgruppe ebenfalls signifikant um $32 \%$ geringer $(p=0,0034)$. Dabei unterschied sich die Wirksamkeit der zweimal täglichen Einnahme nicht von der dreimal täglichen, ergänzte Gold. Das Sicherheitsprofil bezeichnete er nach diesen gepoolten Daten als ,benigne und exzellent“. Ein zu Beginn auftretendes "Flushing" lässt typischerweise nach vier Wochen wieder nach. Die Interimsanalyse der für die Dosis verblindeten Extensionsstudie der Phase-III-Studien [Philipps JT et al. ECTRIMS 2012; Late Breaking Poster P1103] ergab zudem auch über einen längeren Beobachtungszeitraum mit 1.960 ausgewerteten Patientenjahren bisher keine neuen Sicherheitssignale oder Warnhinweise. Gold betonte: „Das ist wichtig: Wir wollen ja frühzeitig und langfristig behandeln, damit die MS bei den meist jungen Patienten auch lange stabil bleibt."

Friederike Klein, freie Medizinjournalistin

Pressekonferenz „Biogen-Idec - Committed to MS Today and Tomorrow", 28. ECTRIMS-Kongress, Lyon, 12.10.2012. Veranstalter: Biogen Idec 